\title{
SLC22A5 polymorphism associated with risk of extra-articular manifestations in rheumatoid arthritis patients
}

\author{
Andrzej Pawlik ${ }^{1}$, Agnieszka Paradowska-Gorycka², Krzysztof Safranow ${ }^{3}$, Violetta Dziedziejko³, \\ Grażyna Dutkiewicz ${ }^{4}$, Sylwia Słucznowska-Głabowska ${ }^{1}$, Zygmunt Juzyszyn ${ }^{5}$, Marek Drozdzik $^{6}$ \\ ${ }^{1}$ Department of Physiology, Pomeranian Medical University, Szczecin, Poland \\ 2Department of Biochemistry and Molecular Biology, National Institute of Geriatrics, Rheumatology and Rehabilitation, Warsaw, Poland \\ ${ }^{3}$ Department of Biochemistry and Medical Chemistry, Pomeranian Medical University, Szczecin, Poland \\ ${ }^{4}$ Department of Nephrology, Transplantology and Internal Diseases, Pomeranian Medical University, Szczecin, Poland \\ ${ }^{5}$ Department of Faculty of Physical Culture and Health Promotion, University of Szczecin, Poland \\ ${ }^{6}$ Department of Experimental and Clinical Pharmacology, Pomeranian Medical University, Szczecin, Poland
}

\begin{abstract}
Objectives: Rheumatoid arthritis (RA), the most common autoimmune disease, is thought to be a complex disease in which a combination of risk alleles from different susceptibility genes predisposes to development of the disease, following exposure to as yet unknown environmental factors. An important component of the carnitine system is the plasma membrane carnitine transporters, also called organic cation transporters, i.e. OCTN1 and OCTN2 encoded by the SLC22A4 and SLC22A5 genes, respectively. The aim of this study was to investigate the association between SLC22A5 polymorphism and RA.

Material and methods: The study was carried out on 404 patients diagnosed with RA according to the criteria of the American College of Rheumatology and 560 healthy subjects. The single nucleotide polymorphism (SNP) within the SLC22A5 gene - 207C>G (rs 2631367) was genotyped using pre-validated TaqMan genotyping assays.

Results: The distribution of SLC22A5 genotypes and alleles in RA patients did not differ significantly from that in healthy controls. Moreover, there were no significant associations between SLC22A5 genotypes and age at time of disease diagnosis, rheumatoid factor, erosive disease and response to treatment with methotrexate. Extra-articular manifestations were diagnosed in 16.7\% of SLC22A5 GG homozygous patients, in $9.4 \%$ with the GC genotype and in $7.2 \%$ of homozygous CC patients. The frequency of extra-articular manifestations was two-fold greater in homozygous GG patients as compared with carriers of the C allele (GG vs. GC + CC), OR $=2.06$ (95\% Cl: 1.11-3.85, $p=0.022$ ). Conclusions: The results of the present study suggest that the SLC22A5 polymorphism may be associated with the development of extra-articular manifestations of RA but the distribution of SLC22A5 genotypes and alleles in studied RA patients did not significantly differ from healthy subjects.
\end{abstract}

Key words: rheumatoid arthritis, SLC22A5, polymorphism, genotypes.

\section{Introduction}

Rheumatoid arthritis (RA), the most common autoimmune disease, is thought to be a complex disease in which a combination of risk alleles from different susceptibility genes predisposes to development of the disease, following exposure to as yet unknown environmental factors. Several genome scans have suggested multiple RA loci [1], and recent case-control association studies have suggested new RA genes [2]. However, only HLADRB1 alleles have

Address for correspondence:

Andrzej Pawlik, Department of Physiology, Pomeranian Medical University, 72 Powstańców Wlkp. St., 70-111 Szczecin, Poland,

e-mail: pawand@poczta.onet.pl

Submitted: 8.11.2018; Accepted: 21.01.2019 
been both linked to and associated with RA, fulfilling the criteria for a fully demonstrated genetic factor [3].

The HLA-DRB1 locus accounts for approximately one-third of genetically determined susceptibility to the disease [4]. The identification of further RA susceptibility loci, both in candidate gene approaches and genome-wide linkage studies, has been hindered in the past by difficulties replicating such results in different study populations.

The role of the carnitine system in cell metabolism is mainly known in the mitochondria, where the interaction between fatty acid and glucose metabolism is fundamental for cell energy production [5]. Adequate carnitine levels are required for normal lipid metabolism and are important for energy metabolism [6]. An important component of the carnitine system is the plasma membrane carnitine transporters, also called organic cation transporters, i.e. OCTN1 and OCTN2 encoded by the SLC22A4 and SLC22A5 genes, respectively. Both genes map to the cytokine gene cluster on chromosome 5 q31 and show $88 \%$ homology and $77 \%$ identity in their sequences. Although OCTN1 and OCTN2 are considered to be carnitine transporters, only OCTN2 is a high-affinity human carnitine transporter, while the carnitine transport activity of OCTN1 is very low [7].

In fact, a recent study has reported that the main substrate of this transporter is ergothioneine, an intracellular antioxidant with metal ion affinity that is transported one hundred times more efficiently than carnitine [8]. OCTN2 is widely expressed in many adult tissues, among them the pancreas, and it participates, at least in part, in proton/organic cation antiport at the renal apical plasma membrane level [9].

Previous reports have revealed the association of some polymorphisms within SLC22A4 and SLC22A5 genes with autoimmune complex diseases, namely RA and Crohn's disease [10,11]. The aim of this study was to investigate the association between SLC22A5 polymorphism - 207C>G (rs 2631367) and RA.

\section{Material and methods}

\section{Patients}

The study was carried out on 404 patients (322 women, 82 men, mean age $57.9 \pm 11.7$ years) diagnosed with RA according to the criteria of the American College of Rheumatology (ACR) [12]. Patients were recruited from the outpatient and inpatient population of the Department of Rheumatology, County Hospital in Szczecin, Poland. All subjects were Caucasians from the Pomerania region of Poland. Subjects enrolled in the study underwent routine biochemical blood analysis and, when required, assays for anticardiolipin anti- bodies, antinuclear antibodies and immunological complexes. X-rays of the chest, hands and feet (erosive or non-erosive RA) were obtained in all patients, and, when necessary, radiographs of other joints. These were interpreted by two different expert radiologists.

The evaluation of subjects included physical examination with particular focus on the pattern of joint involvement, the presence of extra-articular features (such as vasculitis, anemia, sicca syndrome, amyloidosis, organ involvement), and laboratory features such as rheumatoid factor (RF). Amyloidosis was diagnosed by histomorphology (skin and bowel or duodenum biopsy), vasculitis by histomorphology (skin biopsy) and angiogram. The patients were treated with low doses of methotrexate (MTX) and glucocorticosteroids.

The control group consisted of 560 healthy subjects recruited from the Pomerania region (452 women and 108 men, mean age $59.9 \pm 12.6$ years).

The study was approved by the local ethics committee and written informed consent was obtained from all subjects.

\section{Evaluation of treatment efficacy}

Good responders were defined as patients who were receiving MTX and had a DAS28 of $\leq 2.5$ at 6 months of therapy (patients with remission of disease symptoms). Poor responders were defined as patients who were receiving MTX and had a DAS28 of $>2.5$.

\section{Genotyping}

DNA was extracted from $200 \mu$ l of whole blood samples using a GeneMATRIX Quick Blood DNA Purification Kit (EURx, Poland). A SNP within the SLC22A5 gene 207C > G (rs 2631367) was genotyped using pre-validated TaqMan genotyping assays (Life Technologies, USA). Fluorescence data were captured using a 7500 Fast RealTime PCR System (Applied Biosystems, USA).

\section{Statistical analysis}

$\chi^{2}$ or Fisher's exact test was used to compare genotype and allele frequencies between the study groups; $p<0.05$ was considered statistically significant. The age at onset was compared between genotypes using the Kruskal-Wallis test.

\section{Results}

The distribution of SLC22A5 genotypes and alleles in RA patients did not differ significantly from that in healthy controls (Table I). As shown in Table II, there was no significant correlation between age at time of disease diagnosis and the SLC22A5 genotypes. 
Rheumatoid factor was diagnosed in $68.2 \%$ of subjects with SLC22A5 GG genotype, $70.9 \%$ with GC and $68.1 \%$ with CC. These differences were not significant (Table II).

Erosive RA was diagnosed in $87.1 \%$ of GG homozygous patients, in $80.3 \%$ of patients with the GC genotype and $84.1 \%$ of those with CC. These differences were not significant (Table II).

Extra-articular manifestations were diagnosed in $16.7 \%$ of SLC22A5 GG homozygous patients, in $9.4 \%$ with the GC genotype and in $7.2 \%$ of homozygous CC patients. The frequency of extra-articular manifestations was two-fold greater in homozygous GG patients as compared with carriers of the C allele (GG vs. GC + CC), $\mathrm{OR}=2.06(95 \% \mathrm{Cl}: 1.11-3.85, p=0.022)$.

The efficacy of RA therapy with MTX is presented in Table III. Under MTX therapy remission of RA symptoms was achieved in $48.5 \%$ of patients with GG genotype, in $49.3 \%$ of patients with GC genotype and in $46.3 \%$ of patients with CC genotype. The differences were statis tically non-significant.

\section{Discussion}

In the present study we examined the association between SLC22A5 polymorphism and RA. The SLC22A5 genotypes did not correlate significantly with RA susceptibility, age at disease diagnosis, erosive or seropositive disease, or response to treatment with MTX, but were associated with extra-articular manifestations.
Table I. SLC22A5 genotype and allele distribution in rheumatoid arthritis patients and healthy controls

\begin{tabular}{|lcccc|}
\hline Parameters & \multicolumn{2}{c}{ RA patients } & \multicolumn{2}{c|}{ Control group } \\
\cline { 2 - 5 } & $n$ & $\%$ & $n$ & $\%$ \\
\hline GG & 132 & 32.7 & 159 & 28.4 \\
\hline GC & 203 & 50.2 & 288 & 51.4 \\
\hline CC & 69 & 17.1 & 113 & 20.2 \\
\hline G allele & 467 & 57.8 & 606 & 54.1 \\
\hline C allele & 341 & 42.2 & 514 & 45.9 \\
\hline
\end{tabular}

$R A$ - rheumatoid arthritis; no significant differences were noted ( $\chi^{2}$ test).

Organic cation transporters are among a large family of solute carrier transporters that number more than 200 in humans. Three organic cation transporters, SLC22A1 (OCT1), SLC22A2 (OCT2) and SLC22A3 (OCR3), were initially isolated and thereafter two more, SLC22A4 (OCTN1) and SLC22A5 (OCTN2), were isolated as a new family. The first two, SLC22A1 and SLC22A2, seem to transport organic cations in the renal basolateral membrane in a potentially dependent fashion. SLC22A5 is present in various tissues including kidney, skeletal muscle, heart, and placenta [13]. In the kidney SLC22A5 is expressed at the apical membrane of the proximal tubular epithelial cells. Expression was enhanced by tumor necrosis factor (TNF- $\alpha$ ). A murine homologue of SLC22Q4 was found to be expressed in inflamed synovial tissue of collagen-induced arthritis [10].

Table II. Association between SLC22A5 genotypes and clinical rheumatoid arthritis features

\begin{tabular}{|c|c|c|c|c|c|c|c|}
\hline \multirow[t]{2}{*}{ Genotype } & \multirow[t]{2}{*}{$\begin{array}{l}\text { Age of disease } \\
\text { diagnosis }\end{array}$} & \multicolumn{2}{|c|}{ RF } & \multicolumn{2}{|c|}{ Erosive disease } & \multicolumn{2}{|c|}{$\begin{array}{l}\text { Extra-articular } \\
\text { manifestations }\end{array}$} \\
\hline & & $n$ & $\%$ & $n$ & $\%$ & $n$ & $\%$ \\
\hline GG $(n=132)$ & $46.9 \pm 12.8$ & 90 & 68.2 & 115 & 87.1 & 22 & 16.7 \\
\hline $\mathrm{GC}(n=203)$ & $46.9 \pm 13.5$ & 144 & 70.9 & 163 & 80.3 & 19 & 9.4 \\
\hline CC $(n=69)$ & $47.7 \pm 13.0$ & 47 & 68.1 & 58 & 84.1 & 5 & 7.2 \\
\hline$p$-value ${ }^{a}$ & NS & \multicolumn{2}{|c|}{ NS } & \multicolumn{2}{|c|}{ NS } & \multicolumn{2}{|c|}{$0.022^{b}$} \\
\hline
\end{tabular}

$R F$ - rheumatoid factor; NS - not significant; ${ }^{a} \chi^{2}$ test for nominal variables and Kruskal-Wallis test for age of disease diagnosis; ${ }^{b}$ Fisher's exact test for comparison of GG homozygotes vs. C allele carriers (CG +CC); $O R=2.06$ (95\% Cl: 1.11-3.85, $p=0.022$ ).

Table III. Association between SLC22A5 gene polymorphism and response to treatment of rheumatoid arthritis patients with methotrexate

\begin{tabular}{|c|c|c|c|c|c|c|}
\hline \multirow[t]{2}{*}{ SLC22A5 genotype } & \multicolumn{2}{|c|}{$\begin{array}{c}\text { Good responders } \\
n=196\end{array}$} & \multicolumn{2}{|c|}{$\begin{array}{c}\text { Poor responders } \\
n=208\end{array}$} & & \multirow[t]{2}{*}{$p$-value } \\
\hline & $n$ & $\%$ & $n$ & $\%$ & & \\
\hline GG $(n=132)$ & 64 & 48.5 & 68 & 51.5 & $\mathrm{GG}+\mathrm{GC}$ vs. CC & NS \\
\hline $\mathrm{GC}(n=203)$ & 100 & 49.3 & 103 & 50.7 & GG vs. GC + CC & NS \\
\hline CC $(n=69)$ & 32 & 46.3 & 37 & 53.7 & GG vs. CC & NS \\
\hline
\end{tabular}

a Fisher's exact test. 
A mouse strain with a point mutation of the mouse counterpart of human SLC22A5 exhibited an abnormal distribution of carnitine. This point mutation was identified as being involved in a familial carnitine metabolic disorder [14]. SLC22A5 is a physiological transporter of carnitine, and an in vitro analysis revealed that SLC22A5 transports tetraethylammonium (TEA) and carnitine. SLC22A4 and SLC22A5 are 76\% homologous at the amino acid level, and an in vitro investigation of SLC22A4 indicated that it transports carnitine as well as TEA. However, the efficiency of carnitine transportation by SLC22A4 was far lower than that of SLC22A5.

The whole genome linkage study identified IBD5 as an inflammatory bowel disease (IBD)-linked locus [15]. Then the region was further closely evaluated with a dense SNP map that identified haplotypes consisting of two SNPs in SLC22A4 and SLC22A5. One of the SNPs substitutes $503 \mathrm{~L}$ to $\mathrm{F}$ with non-conservative effects on the tertiary structure of SLC22A4 and the other disrupts the heat shock element in the $5^{\prime}$-UTR of SLC22A5. Pharmacological assays revealed that the polymorphic amino acid substitution of SLC22A4 affected the transporting function of the molecule with several changes in $\mathrm{V}_{\text {max }}$ and $\mathrm{K}_{m}$ for some potential transport compounds. The allelic difference in the 5'UTR SNP in SLC22A5 was observed in the binding region of nuclear factors and affected in vitro transcription assays [11]. No conclusion was reached on whether one or both of the genes with the SNPs were responsible for the disease susceptibility.

So far the association between SLC22A5 and RA has not been widely investigated. Previous studies regarding the involvement of the SLC22A4 and SLC22A5 polymorphisms in RA pathogenesis are inconsistent.

Tokuhiro et al. [10] revealed a significant association between RA and the organic cation transporter gene SLC22A4. The authors showed that the expression of SLC22A4 was specific to hematological and immunological tissues, and that SLC22A4 was also highly expressed in the inflammatory joints of mice with collagen-induced arthritis. A SNP affects the transcriptional efficiency of SLC22A4 in vitro, owing to an allelic difference in affinity to runt-related transcription factor 1 (RUNX1), a transcriptional regulator in the hematopoietic system. A SNP in RUNX1 was also strongly associated with RA. These data indicate that the regulation of SLC22A4 expression by RUNX1 is associated with susceptibility to RA, which may represent an example of an epistatic effect of two genes on this disorder.

However, Barton et al. [16] found no evidence for an association between RA and either the SNP or the haplotype previously reported to be associated with RA in a Japanese population by Tokuhiro et al. [10]. These authors suggested that functional polymorphisms of the
OCTN gene locus that have previously been associated with RA in Japanese subjects were not found to be associated with RA in a UK population. The findings do not provide support for a major role of these genes in the etiology of RA in this Caucasian population.

Similarly, Martinez et al. [17] studied whether SLC22A4/SLC22A5 haplotypes are relevant for RA predisposition in a Spanish population. The SLC22A4 and RUNX1 polymorphisms described previously did not show a significant role in RA susceptibility in the above population.

The results of a study by Komlósi et al. [18] suggested no influence of SLC22A4 C6607T genotypic variants on the circulating carnitine ester profile in patients with RA. Frigeni et al. [19] examined the functional properties of the OCTN1 and OCTN2 transporters encoded by SLC22A4 and SLC22A5 genes respectively. The results indicated that the OCTN1 transporter is tolerant of amino acid substitutions in the SLC22A4 gene, whereas the amino acid substitution in the SLC22A5 gene causes reduced transporter activity. Previous studies have shown that the activity of SLC22A5 transport protein in exosomes involved in RA pathogenesis is regulated by the pro-inflammatory cytokine INF- $\gamma[20,21]$.

\section{Conclusions}

The results of the present study indicated that the SLC22A5 polymorphism does not influence RA susceptibility or the response to methotrexate treatment in studied RA patients from a Polish population, but may be associated with the development of extra-articular manifestations of RA.

The authors declare no conflict of interest.

\section{References}

1. Silman AJ, MacGregor AJ, Thomson W, et al. Twin concordance rates for rheumatoid arthritis: results from a nationwide study. Br J Rheumatol 1993; 32: 903-907.

2. Seldin MF, Amos Cl, Ward R, Gregersen PK. The genetics revolution and the assault on rheumatoid arthritis. Arthritis Rheum 1999; 42: 1071-1079.

3. MacGregor AJ, Snieder H, Rigby AS, et al. Characterizing the quantitative genetic contribution to rheumatoid arthritis using data from twins. Arthritis Rheum 2000; 43: 30-37.

4. Wordsworth P, Pile KD, Buckely JD, et al. HLA heterozygosity contributes to susceptibility to rheumatoid arthritis. Am J Hum Genet 1992; 51: 585-591.

5. Ramsay RR. The carnitine acyltransferases: modulators of acyl-CoA-dependent reactions. Biochem Soc Trans 2000; 28: 182-186.

6. Foster DW. The role of the carnitine system in human metabolism. Ann N Y Acad Sci 2004; 1033: 1-16. 
7. Tamai I, Yabuuchi H, Nezu J, et al. Cloning and characterization of a novel human $\mathrm{pH}$-dependent organic cation transporter, OCTN1. FEBS Lett 1997; 419: 107-111.

8. Grundemann D, Harlfinger S, Golz S, et al. Discovery of the ergothioneine transporter. Proc Natl Acad Sci U S A 2005; 102: 5256-5261.

9. Tamai I, Ohashi R, Nezu J, et al. Molecular and functional identification of sodium ion-dependent, high affinity human carnitine transporter OCTN2. J Biol Chem 1998; 273: 20378-20382.

10. Tokuhiro S, Yamada R, Chang $X$, et al. An intronic SNP in a RUNX1 binding site of SLC22A4, encoding an organic cation transporter, is associated with rheumatoid arthritis. Nat Genet 2003; 35: 341-348.

11. Peltekova VD, Wintle RF, Rubin LA, et al. Functional variants of OCTN cation transporter genes are associated with Crohn disease. Nat Genet 2004; 36: 471-475.

12. Felson DT, Anderson JJ, Boers M, et al. The American College of Rheumatology preliminary core set of disease activity measures for rheumatoid arthritis clinical trials. The Committee on Outcome Measures in Rheumatoid Arthritis Clinical Trials. Arthritis Rheum 1993; 36: 729-740.

13. Tamai I, Ohashi R, Nezu Jl, et al. Molecular and functional characterization of organic cation/carnitine transporter family in mice. J Biol Chem 2000; 275: 40064-40072.

14. Nezu J, Tamai I, Oku A, et al. Primary systemic carnitine deficiency is caused by mutations in a gene encoding sodium ion-dependent carnitine transporter. Nat Genet 1999; 21 : 91-94.

15. Rioux JD, Silverberg MS, Daly MJ, et al. Genome wide search in Canadian families with inflammatory bowel disease reveals two novel susceptibility. Am J Hum Genet 2000; 66: 18631870.

16. Barton A, Eyre S, Bowes J, et al. Investigation of the SLC22A4 gene (associated with rheumatoid arthritis in a Japanese population) in a United Kingdom population of rheumatoid arthritis patients. Arthritis Rheum 2005; 52: 752-758.

17. Martinez A, Valdivia A, Pascual-Salcedo D, et al. Role of SLC22A4, SLC22A5, and RUNX1 genes in rheumatoid arthritis. J Rheumatol 2006; 33: 842-846.

18. Komlósi K, Talián GC, Faragó B, et al. No influence of SLC22A4 C6607T and RUNX1 G24658C genotypic variants on the circulating carnitine ester profile in patients with rheumatoid arthritis. Clin Exp Rheumatol 2008; 26: 61-66.

19. Frigeni M, lacobazzi F, Yin X, Longo N. Wide tolerance to amino acids substitutions in the OCTN1 ergothioneine transporter. Biochim Biophys Acta 2016; 1860: 1334-1342.

20. Console L, Scalise M, Indiveri C. Exosomes in inflammation and role as biomarkers. Clin Chim Acta 2019; 488: 165-171.

21. Kato T, Miyaki S, Ishitobi $\mathrm{H}$, et al. Exosomes from IL-1beta stimulated synovial fibroblasts induce osteoarthritic changes in articular chondrocytes. Arthritis Res Ther 2014; 16: R163. 